\title{
The Effect of Repetitive Rugby Scrummaging on Force Output and Muscle Activity
}

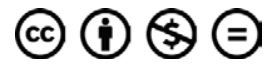

Authors

Darryl J. Cochrane ${ }^{1}$, Keegan Harnett², Nicolas Lopez-Villalobos ${ }^{3}$, Jeremy Hapeta ${ }^{1}$

Affiliations

1 School of Sport \& Exercise, Massey University, Palmerston North, New Zealand

2 Rehab Physio Centre, Palmerston North, New Zealand

3 Institute of Veterinary, Massey University, Animal and Biomedical Sciences, Palmerston North, New Zealand

Key words

isometric, fatigue, electromyography, front row, performance

received 15.01 .2017

revised 24.03.2017

accepted 24.03.2017

\section{Bibliography}

DOI http://dx.doi.org/10.1055/s-0043-108192

Sports Medicine International Open 2017; 1: E89-E93

(C) Georg Thieme Verlag KG Stuttgart · New York

ISSN 2367-1890

Correspondence

Dr. Darryl J. Cochrane

Massey University

School of Sport \& Exercise
Tennent Drive

4442, Palmerston North

New Zealand

Tel.: +64/6/350 5799, Fax: +64/6/3505661

d.cochrane@massey.ac.nz

\section{ABSTRACT}

During rugby scrummaging, front row forwards encounter high levels of force that has been suggested to cause transient fatigue and is likely to reduce subsequent performance. However, little is known about the effect of repetitive scrummaging on force output and onset of fatigue. Twelve male front row forwards $(21.5 \pm 2.3 \mathrm{yr}$; height $185.7 \pm 4.4 \mathrm{~cm}$; body mass $108.5 \pm 7.1 \mathrm{~kg}$ ) each performed three sets of five maximaleffort isometric scrums for $10 \mathrm{~s}$, with $40 \mathrm{~s}$ rest separating each repetition; 2 min recovery was provided between each set. Force output and electromyography (EMG) of the right medial gastrocnemius (MG), biceps femoris (BF), gluteus maximus (GM), erector spinae (ES), rectus abdominis (RA), external oblique (EO), internal oblique (IO), and rectus femoris (RF) were assessed. There was no significant force decrement from performing 15 scrums and no fatigue was detected from EMG median frequency and mean amplitude. For training and practice purposes, coaches and trainers can be confident that 15 individual repetitive static scrums against a machine are unlikely to cause a reduction in force production and promote fatigue. However, the effect of rugbyrelated activities in conjunction with scrummaging requires further research to determine if transient fatigue is causal to scrummaging for subsequent performance.
\end{abstract}

\section{Introduction}

The rugby union scrum is an important set piece used to restart play after a minor infringement. Of the eight players that constitute a scrum per team, the three front row forwards (loose-head prop, hooker, and tight-head prop) are the only players that bind and collide with their opponent's scrum. Considerable forces are exerted on the front row, where the total force generated during machine scrummaging can range from $16.5 \mathrm{kN}$ (elite, forward pack) to $12.0 \mathrm{kN}$ (amateur, forward pack) [15]. Furthermore, single player scrummaging against a scrum machine has shown to generate peak forces of $2.8 \mathrm{kN}$ [4], and 3.1-4.4 kN [20], whilst larger head forces have been reported in tight-head prop and hooker ( 290N), compared to loose-head prop ( 90N) [3] during live 3 v 3 scrummaging.

Front row forwards encounter high levels of static exertion where the intensity rather than the quantity of scrummaging has been suggested to cause transient neuromuscular fatigue [19], which is likely to reduce subsequent performance and may be a predisposing factor for injury [1]. To date, research has focused on determining scrum force of front row forwards and its impact on engagement technique and injury occurrence $[9,16]$, but there is a paucity of research investigating the effect of possible fatigue from repetitive scrummaging. Recent evidence is equivocal with force production from repeated scrummaging showing a significant reduction ( $20 \%$ ) [13] or no change [14]. A reduction in muscle contractility and a decline in voluntary activation of the vastus lateralis has been purported and that both peripheral and central mechanisms may be responsible for scrummaging fatigue [14]. However, previous studies have only examined one or two muscles $[13,14]$ and the activation levels of other muscles during scrummaging in front row forwards warrant further investigation $[4,13,14,20]$. Furthermore, the assessment of lower limb and 'core' muscles, (gluteus maximus, gastrocnemius, and the abdominal complex) have been inadequately examined and little is known 
about the associated effect that repetitive scrummaging may have on muscle activation and neuromuscular fatigue levels. Therefore, the aim of this study was to determine whether repetitive scrummaging reduces force output and causes neuromuscular fatigue as determined by electromyography (EMG) in front row forwards. Based on previous findings $[13,14]$, it was hypothesised that repeated scrummaging would reduce muscle activity of the lower limb with a corresponding decline in force production.

\section{Materials \& Methods}

\section{Participants}

Twelve male (academy, development, and semi-professional) front row forwards ( $\mathrm{n}=6$ loose-head props, $\mathrm{n}=4$ tight-head props, 2 hookers; $21.5 \pm 2.3 \mathrm{yr}$; height $185.7 \pm 4.4 \mathrm{~cm}$; body mass $108.5 \pm 7.1 \mathrm{~kg}$ ) who had completed their competitive season volunteered for the study. Exclusion criteria were lower and upper limb injuries, recent fractures and musculoskeletal conditions. Ethical approval was granted by the University Human Ethics Committee, and written informed consent was obtained from participants. This study was performed in accordance with the ethical standards of the journal [12].

\section{Study design}

Every participant performed a warm-up that included: 5 min treadmill running at a self-selected pace, dynamic stretches, and at least four sub-maximal scrummaging trials. Scrummaging was performed indoors with cleated boots on a synthetic grass surface where players were instructed to place their feet equidistant from a rugby-specific dynamometer (Grunt 3000, E-Type Engineering, Invercargill, New Zealand). The scrum dynamometer is a mobile cart, but for this study it was anchored by a wire cable to a ground plate, which allowed players to place their shoulders against the pads of the cart for sustained pushing. The coefficient of variation of static force generation from the scrum dynamometer has reported values of 2.6-4.4\% [2]. For optimal force generation, players were asked to maintain a hip and knee angle of $120^{\circ}[17,21]$, which was manually checked by goniometer. From the call of the researcher 'crouch-bind-set', the player maximally pushed against the two central pads of the rugby dynamometer ( $>$ Fig. 1). Three sets of five maximal-effort isometric scrums were performed for $10 \mathrm{~s}$, with $40 \mathrm{~s}$ rest separating each repetition, and 2 min recovery was provided between each set. The total number of scrums and scrum time were determined from the average in New Zealand's domestic rugby competition. This was estimated by a frequency count from video match recordings where the number of scrums during a match ranged from 9 to 21 (average 15) with the maximum scrum time (from ball fed to when it exits the scrum) being approximately $10 \mathrm{~s}$ (M Cron, personal communication 28 July 2016). The rest period (work-to-rest ratio) between scrums was similar to previously reported research [13].

The scrum force of each repetition was measured by load cell (Sensortronics, Covina, CA, USA) that was connected to the ground plate, which was attached to the main wire cable of the rugby dynamometer. The load cell was calibrated prior to the player's arrival and the force was sampled at $2000 \mathrm{~Hz}$ by an acquisition system

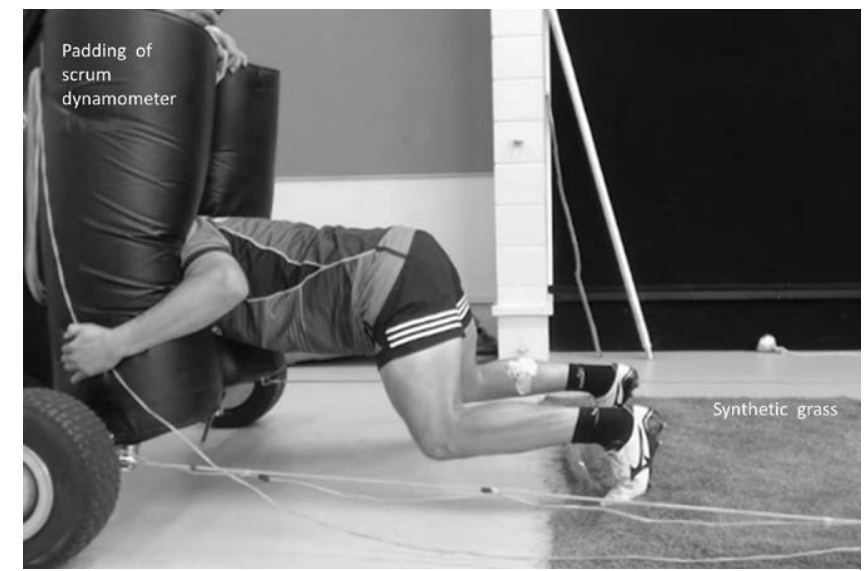

- Fig. 1 Scrum machine positioning.

(Powerlab, 8/30, and Chart v7.1, ADInstruments, Australia) and stored to laptop computer. The force data was subsequently analysed following the initial impact of the player making contact with the rugby dynamometer to the conclusion of 10 s scrummaging.

\section{EMG}

Muscle activity of the right medial gastrocnemius (MG), biceps femoris (BF), gluteus maximus (GM), erector spinae (ES), rectus abdominis (RA), external oblique (EO), internal oblique (IO), and rectus femoris (RF) was assessed using surface EMG. Prior to electrode placement, the area was shaved, gently abraded and cleaned with isopropyl alcohol. Surface pre-gelled $\mathrm{Ag}-\mathrm{AgCl}$ electrodes, $30 \times 22$ mm diameter (Ambu, Ballerup, Denmark) were placed over the mid-belly of the muscle at an inter-electrode distance of $10 \mathrm{~mm}$ parallel to the direction of the fibres [6]. The electrodes were connected to wireless EMG sensors that were securely fastened to the muscle sites with adhesive tape. The sensors pre-amplified the EMG signals using a first-order high-pass filter $(10 \mathrm{~Hz})$ at a gain of 400 . The EMG signals were then transmitted telemetrically in real time to a PC interface-receiver (Telemyo DTS, Noraxon, Scottsdale, AZ, USA) and were recorded by a data acquisition system (MyoResearch XP Master, version 1.07.1, Noraxon, Scottsdale, AZ, USA). Data was filtered with a low-pass filter of $500 \mathrm{~Hz}$ and sampled at $3000 \mathrm{~Hz}$, and was synchronised with video capture (Logitech C920 HD Pro Webcam, NSW, Australia). An external trigger was used to synchronize the load cell and EMG receiver, which embedded an electronic marker into the data files.

The EMG data was analysed by MyoResearch software (XP Master, version 1.07.1, Noraxon, Scottsdale, AZ, USA) where the EMG signals were rectified and smoothed over $50 \mathrm{~ms}$ (root means square algorithm). To determine neuromuscular fatigue, median frequency and mean amplitude were calculated from each scrum, which was defined after the initial impact of the player making contact with the rugby dynamometer to the conclusion of $10 \mathrm{~s}$ of sustained pushing.

\section{Statistical analyses}

All statistical analyses were performed using the Statistical Analysis System software (SAS version 9.3, SAS Institute Inc, Cary, NC, 
USA). Normality testing for force, median frequency (MF) and mean amplitude (MA) was based on the Kolmogorov-Smirnov procedure. The mean amplitude was not normally distributed and the data were transformed using the natural logarithm of the MA $(\log (M A)$. Analyses of variances for the dependent variables were performed using a linear mixed model that included fixed (treatment and time) and random (player) procedures. The model to analyse force included the fixed effect of set and scrum nested within set and the random effect of player to account for repeated measures on the same player. The model to analyse MF and $\log (M A)$ included the fixed effects muscle, set and scrum nested within the interaction muscle-by-set, and the random effect of player to account for repeated measures on the same player.

Least squares means and standard errors for the fixed effects were obtained and used for multiple comparisons using the least significant difference. Differences between means were considered significant at $P \leq 0.05$ and the measure of spread is displayed as \pm standard deviation (SD).

\section{Results}

The mean force of the 15 scrums was $2225.9 \pm 355.5 \mathrm{~N}$. Set 1 reported a mean force of $2237.7 \pm 332.6 \mathrm{~N}$; set $2,2223.2 \pm 314.6 \mathrm{~N}$ and set 3, $2216.8 \pm 416.6 \mathrm{~N}$. There was no main effect of set, such that the mean force from the three sets were similar $(p=0.83)$ and there was no significant difference in mean force between scrums within the sets $(p=0.24)$. However, in set 2 , the mean force of scrum 6 was significantly higher $(p=0.026)$ than scrum 8 . In set 3 , the mean force of scrum 11 was significantly higher than the mean of scrum 13 and 14 ( $p=0.033, p=0.019$, respectively) ( $\vee$ Fig. 2 ).

There was no significant decrement in median frequency ( $\triangleright$ Table 1 ) during the sets $(p=0.79)$, the scrums within the sets $(p=0.99)$, and the muscles during scrummage $(p=1.00)$. There was a main effect of muscle $(p<0.05)$ where the median frequency of MG was larger and RA was smaller compared to the other muscles. For BF and RF, median frequency was larger compared to EO, $\mathrm{ES}, \mathrm{IO}, \mathrm{GM}, \mathrm{RA}$ and the median frequency of $\mathrm{IO}$ was larger than $\mathrm{EO}$, ES, GM, RA.

Results indicate there was no increase in mean amplitude ( $\triangleright$ Table 2 ) during the sets $(p=0.36)$, the scrums within the sets $(p=0.77)$, and the interaction between muscles and scrums $(p=1.00)$. There was a main effect of muscle $(p<0.05)$ where mean amplitude of MG was larger, whilst RA was smaller compared to the other muscles. The mean amplitude of RF was larger than BF, EO, $E S, I O, G M, R A$ and the mean amplitude of ES was larger than BF, $E O, G M$, and RA. For IO, the mean amplitude was larger than GM, EO, RA, whilst GM mean amplitude was larger than EO and RA.

\section{Discussion}

The purpose of this study was to examine if repetitive scrummaging would induce neuromuscular fatigue with a reduction in force output. The main finding indicates that the mean force was similar between the three sets of scrummaging. This is supported by recent research that mean scrummaging forces remained unchanged during a total of six $6 \mathrm{~s}$ of sustained pushing against a dynamic scrum machine [14]. In contrast, Morel et al. [13] reported a sig-

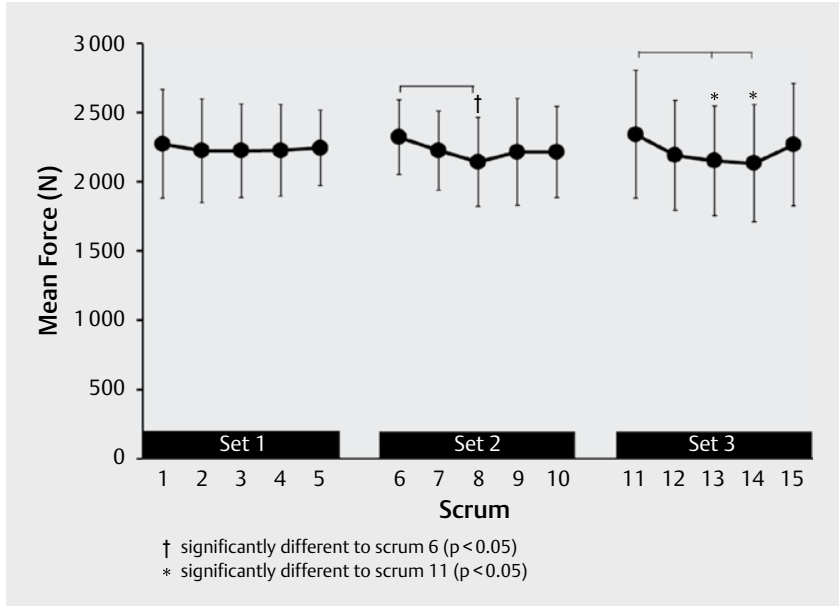

- Fig. 2 † Significantly different to scrum $6(p<0.05) .{ }^{*}$ Significantly different to scrum $11(\mathrm{p}<0.05)$.

nificant reduction ( $23 \%$ ) in force production following five $5 \mathrm{~s}$ scrums. The disparity in results may be due to scrum equipment and protocols [14]. Similarly, in the current study the third scrum in set 2 and 3 (i. e. scrum 8 and 13) generated a lower mean force compared to the initial mean force at the start of those sets (scrum 6 and 11 respectively). Further, the mean force of scrum 11 was significantly higher than scrum 14 but there were no corresponding changes in EMG. Additionally, it is interesting to note that the current front-row forwards generated a mean force of $2200 \mathrm{~N}$ over 15 scrums, which differs from previous values of 1239-1269N [14] and $1340-1420 \mathrm{~N}$ [17]. The different sampling periods (5 s compared to $10 \mathrm{~s}$ ), scrumming equipment, and the physical characteristics of players may explain the discrepancy between results.

Neuromuscular fatigue is defined by a reduction in maximal voluntary force produced by a muscle or muscle groups [7] where an increase in EMG amplitude is thought to reflect the recruitment of additional non-fatigued motor units and/or an increase in motor unit firing frequency [10]. Median frequency is commonly used to detect fatigue from EMG signals [5] to determine the pattern of motor unit activity [11]. Previously, Morel et al. [13] reported a significant reduction (21\%) in EMG (root mean square) of the vastus lateralis following five maximal static scrums lasting $5 \mathrm{~s}$ each. The authors suggested the EMG reduction was likely to involve changes in motor command, spinal inhibition and muscle excitability. Further support of a central mechanism has been purported by recent research where voluntary muscle activation of the knee extensors was reduced during repetitive scrummaging following six $6 \mathrm{~s}$ scrums [14]. Additionally, the same research identified a decline in muscle contractility indicating that peripheral mechanisms also contribute to knee extensor fatigue, although scrummaging force remained unchanged[14]. Nonetheless, the present study did not identify any fatigue effect because no significant changes in median frequency and mean amplitude of the muscles were evident, which is consistent with the force output being maintained.

Previous EMG analysis of scrummaging has been confined to the quadriceps, hamstrings, upper and lower back $[4,13,20]$. In the current study we explored muscles of the kinetic chain, ascending from the ankle to the thoracic spine. To our knowledge, this study 


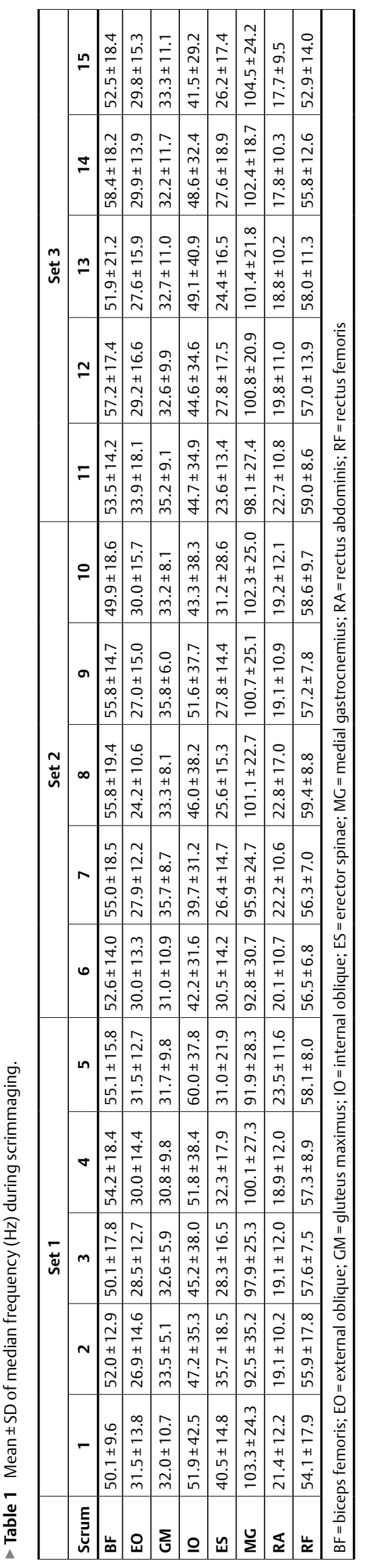

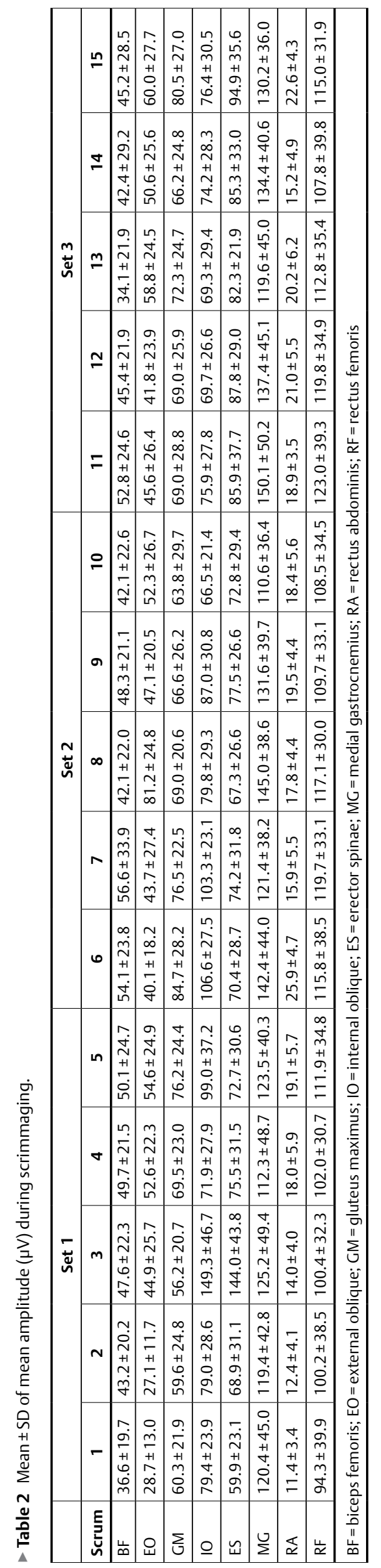


is the first to examine eight muscles during repetitive scrummaging; previously only the vastus lateralis $(\mathrm{VL})$ and erector spine have been assessed $[13,14]$. Earlier research has indicated that the triceps surae is at greater risk of injury from scrummaging [8], although current results indicate MG was activated significantly more. Compared to other muscles it showed no signs of fatigue, with median frequency and mean amplitude remaining unchanged. There was little activation of the abdominal muscles (RA, EO) compared to lower limb muscles (MG, RF) and ES; similiarly BF activation remained low through scrummaging, which is supported by earlier research reporting higher activation levels of VL and ES compared to BF during the pre-engagment phase of scrummaging [20]. The present results are not surprising because machine scrummaging reflects the static nature of the task, which primarily engages the calf and quadricep muscles. However, in live scrummaging front row forwards are exposed to various opposing forces that may produce different activation levels and engagement of the muscles tested.

For this study we decided to focus on force production and muscle activity during repetitive scrummaging; however, this is limited by the fact that it does not include other related front-row forward activities, such as lifting, jogging, sprinting, mauling, rucking, tackling and passing. Therefore, it would be preferable if future studies included other rugby-related aspects to determine if transient fatigue is caused from scrummaging when front-row players move to another rugby-related task [18]. Recently, it has been noted that static machine scrummaging cannot replicate the head forces in live 3 v 3 scrummaging [3], therefore reproducing the opposing forces acting on front row forwards would further improve the ecological validity of prospective studies.

Scrum training and practice have many facets and depending on emphasis (technical, tactical or conditioning), the coach will determine how many scrums will be performed. Often the overload principle is used to induce physiological change to improve performance; however there is a fine line between quantity and quality of scrummaging. According to the current findings, a coach can be confident that 15 individual repetitive static scrums against a machine are unlikely to cause a reduction in force production and promote fatigue. However, some caution is required; the 15 scrums were performed individually, therefore the effect of $3 v 3$ or other derivatives of machine scrummaging, along with live scrummaging is yet to determined. Further, the effect of rugby-related activities to determine if transient fatigue is causal to scrummaging requires additional research.

\section{Conflict of Interest}

The authors declare that they have no conflict of interest.

\section{References}

[1] Best JP, McIntosh AS, Savage TN. Rugby World Cup 2003 injury surveillance project. Br J Sports Med 2005; 39: 812-817

[2] Bramley WJ. The relationship between strength, power and speed measures and playing ability in premier level competition rugby forwards. School of Human Movement Studies, Faculty of Health: Queensland University of Technology; 2006

[3] Burns JA, Seminati E, Cazzola D, Preatoni E, Trewartha G. Machine scrummaging does not mimic the head/neck load experienced in live scrummaging. 2016; In Internet: (22 Nov)

[4] Cazzola D, Stone B, Holsgrove TP, Trewartha G, Preatoni E. Spinal muscle activity in simulated rugby union scrummaging is affected by different engagement conditions. Scand J Med Sci Sports 2016; 26: 432-440

[5] Cifrek M, Medved V, Tonkovic S, Ostojic S. Surface EMG based muscle fatigue evaluation in biomechanics. Clin Biomech 2009; 24: 327-340

[6] Criswell E. Cram's Introduction to surface electromyograhy. 2nd ed. Sudbury, MA, USA: Jones and Bartlett; 2011

[7] Enoka RM, Duchateau J. Muscle fatigue: What, why and how it influences muscle function. J Physiol 2008; 586: 11-23

[8] Flavell CA, Sayers MGL, Gordon SJ, Lee JB. Mechanisms for triceps surae injury in high performance front row rugby union players: $A$ kinematic analysis of scrummaging drills. J Sports Sci Med 2013; 12: 159-164

[9] Fuller CW, Laborde F, Leather RJ, Molloy MG. International Rugby Board Rugby World Cup 2007 injury surveillance study. Br J Sports Med 2008; 42: 452-459

[10] Gandevia SC. Spinal and supraspinal factors in human muscle fatigue. Physiol Rev 2001; 81: 1725-1789

[11] Gerdle B, Larsson B, Karlsson S. Criterion validation of surface EMG variables as fatigue indicators using peak torque - A study of repetitive maximum isokinetic knee extensions. J Electromyogr Kines 2000; 10: 225-232

[12] Harriss DJ, Atkinson G. Ethical standards in sport and exercise science research: 2016 update. Int J Sports Med 2015; 36: 1121-1124

[13] Morel B, Rouffet DM, Bishop DJ, Rota S], Hautier CA. Fatigue induced by repeated maximal efforts is specific to the rugby task performed. Int J Sports Sci Coach 2015; 10: 11-20

[14] Morel B, Hautier CA. The neuromuscular fatigue induced by repeated scrums generates instability that can be limited by appropriate recovery. Scand J Med Sci Sports 2017; 27: 209-216

[15] Preatoni E, Stokes KA, England ME, Trewartha G. The influence of playing level on the biomechanical demands experienced by rugby union forwards during machine scrummaging. Scand J Med Sci Sports 2013; 23: e178-e184

[16] Preatoni E, Stokes KA, England ME, Trewartha G. Engagement techniques and playing level impact the biomechanical demands on rugby forwards during machine-based scrummaging. $\mathrm{Br} J \mathrm{Sports}$ Med 2015; 49: 520-U534

[17] Quarrie KL, Wilson BD. Force production in the rugby union serum. J Sports Sci 2000; 18: 237-246

[18] Quarrie KL, Hopkins WG, Anthony MJ, Gill ND. Positional demands of international rugby union: Evaluation of player actions and movements. J Sci Med Sport 2013; 16: 353-359

[19] Roberts SP, Trewartha G, Higgitt RJ, El-Abd J, Stokes KA. The physical demands of elite English rugby union. J Sports Sci 2008; 26: 825-833

[20] Sharp T, Halaki M, Greene A, Vanwanseele B. An EMG assessment of front row rugby union scrummaging. Int J Perf Anal Spor 2014; 14: 225-237

[21] Wu W-L, Chang J-J, Wu J-H, Guo L-Y. An investigation of rugby scrummaging posture and individual maximum pushing force. J Strength Cond Res 2007; 21: 251-258 